\title{
Late-onset neonatal sepsis in Suzhou, China
}

\author{
Tao Pan ${ }^{1,2+}$, Qiujiao Zhu $^{3+}$, Pei Li ${ }^{1}$, Jun Hua ${ }^{2,3^{*}}$ and Xing Feng ${ }^{1 *}$
}

\begin{abstract}
Background: This study aimed to describe the causative organisms of neonatal late-onset sepsis (LOS) and their antimicrobial resistance in Suzhou, Southeast China over a 7-year period.

Methods: We performed a retrospective study on neonates with LOS from Jan1, 2011 to Dec 31, 2017. The demographic, clinical, and laboratory data of neonates with LOS were analyzed. Logistic regression was used to investigate the risk factors with mortality.

Results: During the study period, 202 neonates with LOS were finally identified. The most common pathogens were Escherichia coli (29.2\%), followed by Klebsiella pneumoniae (19.3\%), and Coagulase-negative Staphylococcus (CoNS) (16.8\%). Nearly $90 \%$ of the K. pneumoniae were resistant to cefazolin and $71.8 \%$ to ceftazidime. Thirty-four patients (16.8\%) died. Multivariable logistic regression showed that significant predictors of mortality were birth weight $<1500 \mathrm{~g}$, respiratory distress and convulsions.
\end{abstract}

Conclusions: Gram-negative organisms have an important role in LOS in our region, with high levels of resistance to third-generation cephalosporins. These data may help in the selection of antibiotics for empirical treatment of neonates with sepsis.

Keywords: Late-onset sepsis, Neonate, Antimicrobial resistance, Mortality

\section{Background}

Neonatal sepsis is an important complication among neonates, particularly those born prematurely [1-4]. The invasive infections can lead to severe morbidities and mortality, and increase the cost of medical care. In 2012, neonatal sepsis lead to 350,000 deaths in South Asia [1]. Neonatal sepsis increases globally because of the increase in premature infants $[5,6]$.. Managing neonatal sepsis is a challenge for physicians.

Depending on the onset age of the disease, neonatal sepsis is divided into early neonatal sepsis and late onset sepsis (LOS). Early neonatal sepsis is mainly due to organisms acquired before and during delivery, whereas

\footnotetext{
*Correspondence: 1970_sz@163.com; feng_xingg@163.com

${ }^{\dagger}$ Tao Pan and Qiujiao Zhu contributed equally to this work.

${ }^{2}$ Department of Internal Medicine, Children's Hospital of Wujiang District, Suzhou, China

'Department of Neonatology, Children's Hospital of Soochow University, Soochow University, Suzhou, China

Full list of author information is available at the end of the article
}

LOS is due to organisms acquired after delivery from nosocomial or community sources. In the past few decades, the mortality rate of LOS has remained at a high level (5-15\%) in most neonatal care facilities [7-9]. Coagulase-negative Staphylococcus (CoNS) is reported as the main pathogen of LOS [10-12]. However, the spectrum of pathogens varies among different regions and may change over time in the same place [13, 14]. The investigation of organisms causing LOS and the monitoring of their antimicrobial sensitivity can be used to guide appropriate empirical treatment and help develop cost effective interventions to prevent neonatal deaths.

China is known to be among the highest antibiotic consumers all over the world $[15,16]$. However, there is no national wide survey about the pathogen distribution and their antimicrobial sensitivity in patients with LOS. This study aimed to describe the causative organisms of LOS and their antimicrobial resistance in Suzhou,

(c) The Author(s). 2020 Open Access This article is licensed under a Creative Commons Attribution 4.0 International License, which permits use, sharing, adaptation, distribution and reproduction in any medium or format, as long as you give appropriate credit to the original author(s) and the source, provide a link to the Creative Commons licence, and indicate if changes were made. The images or other third party material in this article are included in the article's Creative Commons licence, unless indicated otherwise in a credit line to the material. If material is not included in the article's Creative Commons licence and your intended use is not permitted by statutory regulation or exceeds the permitted use, you will need to obtain permission directly from the copyright holder. To view a copy of this licence, visit http://creativecommons.org/licenses/by/4.0/ The Creative Commons Public Domain Dedication waiver (http://creativecommons.org/publicdomain/zero/1.0/) applies to the data made available in this article, unless otherwise stated in a credit line to the data. 
Southeast China over a 7-year period. Risk factors with mortality were also investigated.

\section{Materials and methods Study design}

We performed a retrospective study of LOS among neonates who were hospitalized in the neonatal intensive care unit (NICU) in the Children's Hospital of Soochow University from Jan1, 2011 to Dec 31, 2017. The medical records of all neonates in the NICU were reviewed by two neonatologists. Children's Hospital of Soochow University is a 1300-bed teaching hospital in southeast China that provides primary to tertiary care for children younger than 18 years old. This hospital has a NICU with a total of 50 beds. This study was approved by the Medical Ethics Committee of Children's Hospital of Soochow University.

\section{Definition}

LOS was defined as the growth of a single potentially pathogenic organism (bacterium or fungus) from blood or cerebrospinal fluid (CSF) in neonates $>3$ days of age with clinical and laboratory findings consistent with infection [17]. Infants with only one positive blood culture for a common skin contaminant (e.g., diphtheroids, Bacillus, Propionibacterium, CoNS, or micrococci) without any symptoms of sepsis were excluded due to the possibility of contamination during sample handling. Blood and CSF Cultures and the identification of pathogens were performed using the VITEK $^{\circ} 2$ automated system (BioMérieux, France). Antibiotic susceptibility tests and the identification of ESBL were performed using antibiotic susceptibility test cards (VITEK $^{\circ} 2$ automated system).

\section{Data collections}

Standardized forms were used to describe the maternal and neonatal characteristics for each infection case (antenatal screening cultures, mode of delivery, neonatal clinical signs and symptoms, age of onset, therapies, and outcomes).

\section{Statistics}

Characteristics, clinical symptoms, and laboratory parameters of infants with LOS were described with data expressed as numerical values with percentage or mean and standard deviation as appropriate. Clinical symptoms were compared between preterm and term infants with LOS and characteristics compared between infants who died versus survived. Unadjusted comparisons were assessed by Fisher's exact or chi-square test. A logistic regression model that included variables significant at $p<0.1$ in univariate comparisons between infants who died and survived was used to examine adjusted associations with mortality. Adjusted odds ratios (OR) with 95\% confidence intervals (CI) were reported from this model. Statistical analyses were performed using the Statistical Package for the Social Sciences (version 17.0).

\section{Results}

\section{Demographics and clinical characteristics}

During the study period, 5522 infants admitted to the NICU in Children's Hospital of Soochow University were identified. Of these, a total of 213 neonates with suspected cases of sepsis were enrolled. Eleven cases were excluded because their cultures were considered contaminated. As a result, 202 neonates with LOS were finally identified. The demographic and clinical characteristics of the neonates are shown in Table 1. Of the patients, $84(41.6 \%)$ cases were female and 118 (58.4\%) were male. The mean birth weight was $2132 \pm 719 \mathrm{~g}$. The mean gestational age was $33.6 \pm 4.2$ weeks. The majority of infants with LOS were preterm (154/202, $76.2 \%)$ while about a quarter were term (48/202, 23.8\%). Term infants present with clinical symptoms of sepsis at the time of admission or who developed sepsis during hospitalization were admitted to the NICU. The mean postnatal age at diagnosis was $13 \pm 7$ days. Fever (160/ 202, 79.2\%), respiratory distress $(118 / 202,58.4 \%)$, neonatal jaundice $(114 / 202,56.4 \%)$ were the most common clinical manifestations. Respiratory distress (122 [79.2\%] vs 25 [52.1\%]) and feeding intolerance (115 [74.7\%] vs 22 [45.8\%]) were more common in preterm than in term neonates (both $P<0.01$ ). There were no significant differences between preterm and term neonates in terms of fever (122 [79.2\%] vs 38 [79.2\%]), neonatal jaundice (102 [66.2\%] vs $36[75.0 \%])$ and convulsions (16 [10.4\%] vs 6 [12.5\%], all $P>0.05)$.

\section{Etiologic pathogens}

Among the 202 LOS, 86 (42.6\%) episodes were caused by Gram-positive organisms; 104 (51.5\%) episodes, by Gram-negative organisms; and 12 (5.9\%) episodes, by Candida albicans. The most common pathogens were Escherichia coli (29.2\%), followed by Klebsiella pneumoniae (19.3\%), and CoNS (16.8\%) (Table 2).

Of all the 202 LOS cases, $149(73.8 \%)$ received a lumbar puncture, and 32 (15.8\%) of the CSF samples were positive. Of the 32 positive CSF cultures, five were from neonates with a negative blood culture. The other 27 infants have the same organisms isolated on both blood and CSF. K. pneumoniae, E. coli, and CoNS were the most common organisms isolated from the CSF of children with LOS.

We also compared the distribution of organisms between 2011 and 2013 and 2014-2017 (Fig. 1). During 2011-2013, CoNS was the most frequent cause of LOS (24.7\%), followed by E. coli (22.7\%) and K. pneumoniae 
Table 1 Demographic and Clinical Characteristics of the 202 Cases with Late Onset Sepsis

\begin{tabular}{ll}
\hline Characteristics & Number (\%) \\
\hline Sex & \\
Male & $118(58.4)$ \\
Female & $84(41.6)$ \\
Gestational age $(w k)$ & \\
$<28$ & $26(12.9)$ \\
$28-31$ & $31(15.3)$ \\
$32-36$ & $97(48.0)$ \\
$\geq 37$ & $48(23.8)$ \\
Birth weight (g) & \\
$<1500$ & $48(23.8)$ \\
1500-<2500 & $93(46.0)$ \\
$2500-<4000$ & $41(20.3)$ \\
$\geq 4000$ & $20(9.9)$ \\
Cesarean delivery & $125(61.9)$ \\
Multiple gestation & $9(4.6)$ \\
Membrane rupture $\geq 18 \mathrm{~h}$ before delivery & $69(34.2)$ \\
Exposure to intrapartum antibiotics & $53(26.2)$ \\
Pregestational diabetes mellitus & $21(10.4)$ \\
Maternal intrapartum fever & $32(15.8)$ \\
Patients with underlying conditions ${ }^{a}$ & $24(11.9)$ \\
Clinical symptoms and signs & $160(79.2)$ \\
Fever & $118(58.4)$ \\
Nespiratory distress & $114(56.4)$ \\
Coeding intolerance & $108(53.5)$ \\
& $22(10.9)$ \\
&
\end{tabular}

Laboratory parameters

White blood cell counts $(<8$ or $>12) 10^{9} / \mathrm{L}$

$166(82.2)$

Hemoglobin $(<110$ or $>160) \mathrm{g} / \mathrm{L}$

$141(69.8)$

Platelet counts $(<100$ or $>300) 10^{9} / \mathrm{L}$

38 (18.8)

Procalcitonin $(\geq 0.05) \mathrm{ng} / \mathrm{mL}$

$198(98.0)$

C-reactive protein $(\geq 8) \mathrm{mg} / \mathrm{L}$

$153(76.2)$

${ }^{a}$ Among the 24 patients, 22 had congenital heart disease, 1 had congenital biliary atresia and 1 had intestinal duplication

(16.5\%). In 2014-2017, E. coli was the most frequent cause of LOS (35.2\%), followed by $K$. pneumoniae (21.9\%) and CoNS (7.6\%).

\section{Antibiotic susceptibility}

All of the tested E. coli isolates (58/58) were sensitive to amikacin, nitrofurantoin, piperacillin/ tazobactam, cefoperazone/sulbactam, and imipenem (Table 3). Among the tested $K$. pneumoniae isolates, all (39/39) were sensitive to amikacin, piperacillin/tazobactam, cefoperazone/ sulbactam and imipenem, whereas nearly $90 \%$ were resistant to ampicillin (35/39) and cefazolin (34/39), $71.8 \%(28 / 39)$ to ceftazidime and $38.4 \%(15 / 39)$ to nitrofurantoin. Enterobacteriaceae isolates, including E. coli, $K$. oxytoca, and $K$. pneumoniae were also evaluated for extended-spectrum beta-lactamase (ESBL). Finally, $74.4 \%(29 / 39)$ of the $K$. pneumoniae isolates and $5.1 \%$ (3/59) of E. coli isolates were identified as ESBL Enterobacteriaceae. Among the tested ESBL Enterobacteriaceae isolates, all (32/32) were sensitive to piperacillin/tazobactam, cefoperazone/sulbactam; however, all were resistant to cefazolin and $78.1 \%(25 / 32)$ to ceftazidime. Among the tested CoNS isolates, all (34/34) were sensitive to vancomycin, $70.6 \%(24 / 34)$ were sensitive to cefoperazone/sulbactam, whereas $67.6 \%(23 / 34)$ were resistant to ampicillin and $61.8 \%(21 / 34)$ were resistant to cefazolin.

\section{Infant antibiotic therapy}

All of the neonates were treated with antibiotics. The majority of the neonates with infections $(80.2 \%)$ were started on cephalosporins (first or third generation), while $18.8 \%$ received amoxicillin. After identifying the pathogen and its antibiotic susceptibility profile, antibiotics were changed for $43.6 \%$ of the neonates. Cefoperazone sulbactam, vancomycin and meropenem were the most frequently substituted antibiotics. Among the 86 neonates with Gram-positive bacterial infections, the majority, $65.1 \%$, continued on cephalosporins or amoxicillin once the culture results were available, whereas $22.1 \%$ were switched to vancomycin with or without continuation of the initially-prescribed antibiotics. Among the 104 neonates who tested positive for Gramnegative organisms, $53.8 \%$ had their initial antibiotic therapies continued, whereas $37.5 \%$ were switched to meropenem or cefoperazone sulbactam.

\section{Mortality}

Most neonates (83.2\%) with LOS survived to hospital discharge, while $34(16.8 \%)$ patients died; E. coli was responsible for nearly one-third $(11 / 34,32.4 \%)$ of deaths and CoNS responsible for $17.6 \%(6 / 34)$. The proportion of infants with birth weight $<1500 \mathrm{~g}$ was larger among infants who died compared to infants who survived $(64.7 \%$ vs $15.5 \%, p<0.001)$ as was the proportion born at gestational age $<32$ weeks $(55.9 \%$ vs $22.6 \%, \mathrm{p}<0.001)$ (Table 4). Respiratory distress, neonatal jaundice, and convulsions were also more frequently experienced by infants who died. Statistically significant differences between infants who died versus survived were not observed for the other characteristics examined, including the proportion with $E$. coli infection. When the 5 variables significant in univariate analysis were included in a logistic regression model, birth weight $<1500 \mathrm{~g}$ (OR:3.38; 95\% CI: 1.33-8.62), respiratory distress (OR:4.25; 95\% CI: 1.31-13.77), and convulsions (OR:4.09; 95\% CI: 
Table 2 The Microbiology Identified in 202 Neonatal Patients with Late Onset Sepsis in Suzhou, China

\begin{tabular}{llll}
\hline Pathogens & Total $n(\%)$ & Preterm $n(\%)$ & Term $n(\%)$ \\
\hline \multicolumn{1}{c}{ Gram negative bacteria } & $104(51.5)$ & $83(53.9)$ & $21(43.8)$ \\
Escherichia coli & $59(29.2)$ & $49(31.8)$ & $10(20.8)$ \\
Klebsiella pneumoniae & $39(19.3)$ & $30(19.5)$ & $9(18.8)$ \\
Klebsiella oxytoca & $3(1.5)$ & $2(1.3)$ & $1(2.1)$ \\
Enterobacter aerogenes & $3(1.5)$ & $2(1.3)$ & $1(2.1)$ \\
Gram positive bacteria & $86(42.6)$ & $59(38.3)$ & $27(56.3)$ \\
Coagulase-negative staphylococci & $34(16.8)$ & $23(14.9)$ & $11(22.9)$ \\
Staphylococcus aureus & $17(8.4)$ & $10(6.5)$ & $7(14.6)$ \\
Enterococcus faecalis & $15(7.4)$ & $11(7.1)$ & $4(8.3)$ \\
Group B Streptococcus & $15(7.4)$ & $11(7.1)$ & $4(8.3)$ \\
Listeria monocytogenes & $5(2.5)$ & $4(2.6)$ & $1(2.1)$ \\
\multicolumn{1}{c}{ Fungi } & $12(5.9)$ & $12(5.9)$ & 0 \\
\hline
\end{tabular}

1.36-12.31) remained significantly associated with mortality (Table 5).

\section{Discussion}

This study aimed to investigate the prevalence of culture-proven LOS in Suzhou, Southeast China and to identify the main causative organisms of LOS. Among 202 culture-proven LOS, E. coli was the most commonly isolated bacteria, followed by K. pneumoniae and CoNS.

Previous studies have revealed that gram-positive bacteria were the main causative organisms, with CoNS responsible for the most common organism of LOS [18, 19]. Majeda et al. reported that CoNS alone was responsible for more than one-third of LOS cases in Arab states in the Gulf region [20]. Matthew et al. found that for nearly 20 years, CoNS was the organism most responsible for late-onset sepsis in their NICU [21]. In some regions from developing countries, CoNS have been reported to play a less important role [22, 23]. In China, Guo et al. found that CoNS was the third common organism responsible for LOS in South China [24], while $\mathrm{Li}$ et al. found that CoNS was the most common organism in Southeast China [25]. The results in our study are in line with Guo's study. Thus, the role of CoNS responsible for LOS are likely to vary in different regions in China. Despite the importance of CoNS as etiological agents of LOS, determining whether CoNS isolates are true pathogens or contaminants is still very

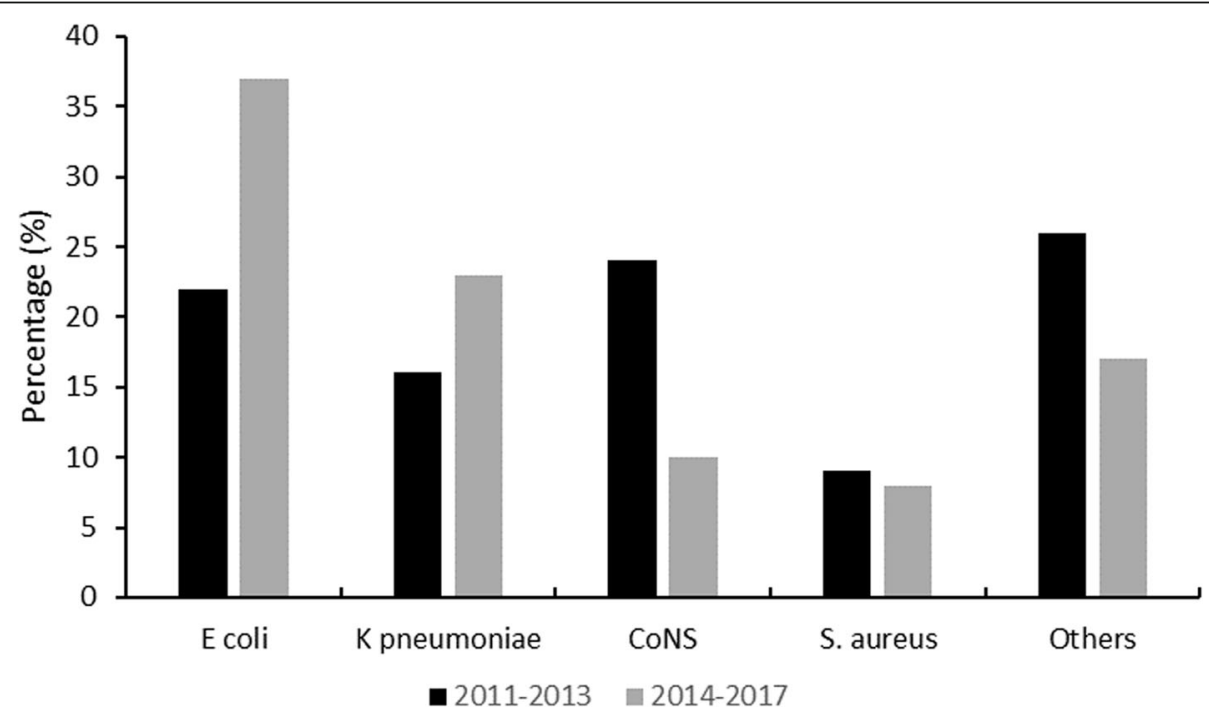

Fig. 1 Distribution of the organisms of late onset infections between 2011 and 2013 and 2014-2017 
Table 3 Selected antimicrobial susceptibility patterns from the main bacteria of septic neonates, 2011 to 2017

\begin{tabular}{llll}
\hline \multicolumn{1}{c}{ Antibiotics } & \multicolumn{3}{c}{ No. (\%) susceptible } \\
\cline { 2 - 4 } & Coagulase-negative staphylococci $(n=34)$ & Escherichia coli $(n=58)^{{ }^{a}}$ & Klebsiella pneumoniae $(n=39)$ \\
\hline Amoxicillin & $11(32.4)$ & - & $39(100)$ \\
Amikacin & - & $58(100)$ & $4(10.3)$ \\
Ampicillin & $11(32.4)$ & $13(22.4)$ & $5(12.8)$ \\
Cefazolin & $13(38.2)$ & $26(44.8)$ & $11(28.2)$ \\
Ceftazidime & $22(64.7)$ & $49(84.5)$ & $39(100)$ \\
Cefoperazone/sulbactam & $24(70.6)$ & $58(100)$ & $23(59.0)$ \\
Furadantin & $34(100)$ & $58(100)$ & $39(100)$ \\
Imipenem & - & $58(100)$ & $33(84.6)$ \\
Ciprofloxacin & $27(79.4)$ & $25(43.1)$ & $24(61.6)$ \\
Nitrofurantoin & $34(100)$ & $58(100)$ & $39(100)$ \\
Piperacillin/tazobactam & - & $58(100)$ & $26(66.7)$ \\
Sulfamethoxazole-trimethoprim & $17(50.0)$ & $19(32.8)$ & - \\
Vancomycin & $34(100)$ & - & \\
\hline
\end{tabular}

${ }^{a}$ Information on antibiotic sensitivity was available for 58 of the 59 neonates with Escherichia coli sepsis

challenging $[21,26]$. In our study, CoNS were considered pathogenic only if, in addition to a positive blood culture, there were clinical and laboratory findings to support infection. Furthermore, 6 died from LOS by CoNS, suggesting that these were true infections.

E. coli and K. pneumoniae were the most common isolated Gram-negative bacteria in our study, accounting for 29.2 and $19.3 \%$ of all the isolated pathogens, respectively. Other Gram-negative bacilli were recovered but in a few numbers. The predominance of $E$. coli and $K$. pneumoniae among the causative Gram-negative pathogens was also reported in other studies in China and other different countries [24, 27]. In contrast,
Enterobacter was reported as the most common Gramnegative isolates in other studies [20,28].

Fungi are also significant pathogens of neonatal sepsis, especially in preterm and low birth weight infants. Fungi infections are usually acquired during prolonged hospital stay of preterm neonates [29]. In our study, Candida spp. accounted for $5.9 \%$ of isolated pathogens. All the patients with Candida spp. infection were preterm low birth weight infants. The fungi infection rate in our study is similar to the results of Guo's study [24], but significantly higher than the results of 2 other studies conducted in other regions of China [13, 14].

In our study, more than $70 \%$ of the patients received lumbar puncture. and $15.8 \%$ of the CSF samples were

Table 4 Mortality Among Infants with Late-onset Sepsis

\begin{tabular}{|c|c|c|c|}
\hline Characteristics & Died $(n=34), n(\%)$ & Survived $(n=168), n(\%)$ & $P$ value \\
\hline Birth weight $<1500 \mathrm{~g}$ & $22(64.7)$ & $26(15.5)$ & $<.001$ \\
\hline Gestational age $<32$ wk & $19(55.9)$ & $48(22.6)$ & $<.001$ \\
\hline Male & $19(55.9)$ & $99(58.9)$ & 0.74 \\
\hline Cesarean delivery & $21(61.7)$ & $97(57.7)$ & 0.66 \\
\hline Multiple gestation & $3(6.8)$ & $9(3.4)$ & 0.72 \\
\hline Pregestational diabetes mellitus & $7(15.9)$ & $31(11.8)$ & 0.45 \\
\hline Maternal intrapartum fever & $9(20.1)$ & $48(18.3)$ & 0.74 \\
\hline E coli infection & $11(32.4)$ & $48(28.8)$ & 0.67 \\
\hline \multicolumn{4}{|l|}{ Clinical symptoms and signs } \\
\hline Fever & $24(70.6)$ & $136(80.9)$ & 0.18 \\
\hline Respiratory distress & $30(88.2)$ & $88(52.3)$ & 0.001 \\
\hline Neonatal jaundice & $12(35.3)$ & $102(60.7)$ & 0.01 \\
\hline Feeding intolerance & $22(64.7)$ & $86(51.2)$ & 0.15 \\
\hline Convulsions & $8(23.5)$ & $14(8.3)$ & 0.01 \\
\hline
\end{tabular}


Table 5 Mortality Among Infants with Late-onset Sepsis

\begin{tabular}{|c|c|c|c|}
\hline Characteristics & Died n/N (\%) & $P$ value & Adjusted OR for death $(95 \% \mathrm{Cl})$ \\
\hline \multicolumn{4}{|c|}{ Birth weight < $1500 \mathrm{~g}$} \\
\hline Y & $22 / 48(45.8)$ & $<0.01$ & $3.38(1.33-8.62)$ \\
\hline $\mathrm{N}$ & $12 / 154(7.8)$ & & 1.0 \\
\hline \multicolumn{4}{|c|}{ Respiratory distress } \\
\hline Y & $30 / 118(25.4)$ & $<0.01$ & $4.25(1.31-13.77)$ \\
\hline N & 4/84 (4.8) & & 1.0 \\
\hline \multicolumn{4}{|l|}{ Convulsions } \\
\hline Y & $8 / 22(36.4)$ & $<0.01$ & $4.09(1.36-12.31)$ \\
\hline $\mathrm{N}$ & $26 / 180(14.4)$ & & 1.0 \\
\hline \multicolumn{4}{|c|}{ Gestational age $<32$ wk } \\
\hline Y & 19/67 (28.4) & 0.09 & $0.40(0.14-1.17)$ \\
\hline $\mathrm{N}$ & 15/135 (11.1) & & 1.0 \\
\hline \multicolumn{4}{|c|}{ Neonatal jaundice } \\
\hline Y & $12 / 114(10.5)$ & 0.52 & $0.74(0.30-1.84)$ \\
\hline $\mathrm{N}$ & $22 / 88(25.0)$ & & 1.0 \\
\hline
\end{tabular}

positive. Neonatal meningitis remains a substantial cause of sepsis-related morbidity and mortality in neonates. Other than CSF cultures, there are no clinical parameters that excludes the diagnosis of meningitis in neonates [30]. Hoque et al. also reported that it is important to do a lumbar puncture for all suspected septicemia cases in neonates. They found that the clinical manifestations were similar in both sepsis and meningitis cases, while mortality was high among the meningitis cases compared with those having sepsis alone $(37.5 \%$ vs. 13.3\%) [31].

Because LOS is usually caused by nosocomially acquired organisms, it has been suggested that resistance to antibiotics is common in LOS pathogens. As the second common pathogen responsible for LOS, nearly $90 \%$ were resistant to cefazolin, $71.8 \%$ to ceftazidime. The high level of resistance of $K$. pneumoniae to thirdgeneration cephalosporins has been reported from developing countries [23, 32]. Among the tested CoNS isolates, all were sensitive to vancomycin. Vancomycin is the most effective and economical drug for treatment of CoNS LOS. However, great concern has been raised due to the widely used vancomycin and the emergence of vancomycin-resistant Staphylococcus [33, 34]. Actually, in our study, about $70 \%$ of the CoNS isolates were sensitive to cefoperazone/sulbactam. We suggested that vancomycin use should not be empirical but based on the results of blood cultures in our region. In China, there are currently no national antimicrobial guidelines for neonatal sepsis. The development of guidelines could be assisted by the findings of our study on both epidemiology and antimicrobial use. Local epidemiology and antimicrobial susceptibility patterns might explain the absence of national guidelines. In our region, the current resistance profile of pathogens makes empiric coverage with cefoperazone/sulbactam seem appropriate.

Previous studies reported that appropriate empiric treatment significantly reduced the duration of treatment [26]. A study by Chiu et al. also showed that appropriate empiric treatment of suspected CoNS LOS significantly reduced the use of vancomycin [35]. As reported by Kaufman, accuracy in diagnosis and use of antimicrobial agents are important to prevent neonatal mortality related to sepsis [36]. In our study, $65.1 \%$ of the neonates who tested positive for Gram-positive organisms and $53.8 \%$ of the neonates who tested positive for Gram-negative organisms had their initial antibiotic therapies continued. Whether appropriate empiric treatment reduced the duration of treatment in our region need further investigations.

In our study, The case-fatality was $16.8 \%$, which is similar to those reported from Asian countries (16\%) $[10,20]$. E. coli were responsible for about one-third (11/ 34 ) of deaths due to LOS. E coli has been found to be associated with most LOS deaths, primarily because of its predominance among very low birth weight infants [21]. However, in our cohort of mostly preterm infants $E$. coli infection was not an independent predictor of mortality. We found that significant predictors of mortality were birth weight $<1500 \mathrm{~g}$, respiratory distress and convulsions. Thus, clinicians should pay more attentions to the low birth weight infants presented with respiratory distress and convulsions.

In conclusion, Gram-negative organisms have an important role in LOS in our region, with high levels of resistance to third-generation cephalosporins. These data may help in the selection of antibiotics for empirical treatment of neonates with sepsis.

\section{Abbreviations}

LOS: Late onset sepsis; CoNS: Coagulase-negative Staphylococcus; CSF: Cerebrospinal fluid; ESBL: Extended-spectrum beta-lactamase

\section{Acknowledgements}

The authors thank all nursing staff working in our department for keeping extremely detailed patient records, which contributed greatly to the completion of this research.

\section{Authors' contributions}

TP and QJZ wrote the main manuscript text. JH and XF conceptualized and designed the study, drafted the initial manuscript, and approved the final manuscript. QJZ and PL carried out the initial analyses, reviewed and revised the manuscript, and approved the final manuscript. All authors read and approved the final manuscript.

\section{Funding}

None.

\section{Availability of data and materials}

The manuscript detailing where the data supporting the findings in this study can be found if requested. 


\section{Ethics approval and consent to participate}

The study was approved by the Medical Ethics Committee of Soochow University. The parents of all study participants gave both verbal and written informed consent before study enrollment.

\section{Consent for publication}

Not applicable.

\section{Competing interests}

The authors declare that they have no competing interests.

\section{Author details}

'Department of Neonatology, Children's Hospital of Soochow University, Soochow University, Suzhou, China. ${ }^{2}$ Department of Internal Medicine, Children's Hospital of Wujiang District, Suzhou, China. ${ }^{3}$ Department of Emergence, Children's Hospital of Soochow University, Soochow University, Suzhou, China.

Received: 15 December 2019 Accepted: 28 April 2020

Published online: 29 May 2020

\section{References}

1. Seale AC, Blencowe H, Manu AA, Nair H, Bahl R, Qazi SA, Zaidi AK, et al. Estimates of possible severe bacterial infection in neonates in sub-saharan africa, south asia, and latin america for 2012: a systematic review and metaanalysis. Lancet Infect Dis. 2014;14:731-41.

2. Hibberd PL, Hansen NI, Wang ME, Goudar SS, Pasha O, Esamai F, Chomba E, et al. Trends in the incidence of possible severe bacterial infection and case fatality rates in rural communities in sub-saharan africa, south asia and latin america, 2010-2013: a multicenter prospective cohort study. Reprod Health. 2016;13:65.

3. Stoll BJ, Hansen NI, Bell EF, Walsh MC, Carlo WA, Shankaran S, Laptook AR, et al. Trends in care practices, morbidity, and mortality of extremely preterm neonates, 1993-2012. JAMA. 2015;314:1039-51.

4. Stoll BJ, Hansen NI, Bell EF, Shankaran S, Laptook AR, Walsh MC, Hale EC et al. Neonatal outcomes of extremely preterm infants from the nichd neonatal research network. Pediatrics. 2010;126:443-56.

5. Beck S, Wojdyla D, Say L, Betran AP, Merialdi M, Requejo JH, Rubens C, et al. The worldwide incidence of preterm birth: a systematic review of maternal. mortality and morbidity. Bull World Health Organ. 2010;88:31-8.

6. Callaghan WM, MacDorman MF, Rasmussen SA, Qin C, Lackritz EM. The contribution of preterm birth to infant mortality rates in the United States. Pediatrics. 2006;118:1566-73.

7. Babazono A, Kitajima H, Nishimaki S, Nakamura T, Shiga S, Hayakawa M, Tanaka T, et al. Risk factors for nosocomial infection in the neonatal intensive care unit by the japanese nosocomial infection surveillance (janis). Acta Med Okayama. 2008;62:261-8.

8. Kung YH, Hsieh YF, Weng YH, Lien Rl, Luo J, Wang Y, Huang YC, et al. Risk factors of late-onset neonatal sepsis in Taiwan: a matched case-control study. J Microbiol Immunol Infect. 2016;49:430-5.

9. Stoll BJ, Hansen N, Fanaroff AA, Wright LL, Carlo WA, Ehrenkranz RA, Lemons JA, et al. Late-onset sepsis in very low birth weight neonates: the experience of the nichd neonatal research network. Pediatrics. 2002;110: 285-91.

10. Al-Taiar A, Hammoud MS, Cuiqing L, Lee JK, Lui KM, Nakwan N, Isaacs D. Neonatal infections in China, Malaysia, Hong Kong and Thailand. Arch Dis Child Fetal Neonatal Ed. 2013:98:F249-55.

11. Hammoud MS, Al-Taiar A, Thalib L, Al-Sweih N, Pathan S, Isaacs D. Incidence, aetiology and resistance of late-onset neonatal sepsis: a five-year prospective study. J Paediatr Child Health. 2012;48:604-9.

12. Gkentzi D, Kortsalioudaki C, Cailes BC, Zaoutis T, Kopsidas J, Tsolia M, Spyridis N, et al. Epidemiology of infections and antimicrobial use in greek neonatal units. Arch Dis Child Fetal Neonatal Ed. 2019;104:F293-F7.

13. Jiang $Y$, Kuang L, Wang H, Li L, Zhou W, Li M. The clinical characteristics of neonatal sepsis infection in Southwest China. Intern Med. 2016;55:597-603.

14. Zhou B, Liu X, Wu JB, Jin B, Zhang YY. Clinical and microbiological profile of babies born with risk of neonatal sepsis. Exp Ther Med. 2016;12:3621-5.

15. Heddini A, Cars O, Qiang S, Tomson G. Antibiotic resistance in China--a major future challenge. Lancet. 2009;373:30.
16. Zhang R, Eggleston K, Rotimi V, Zeckhauser RJ. Antibiotic resistance as a global threat: evidence from China, Kuwait and the United States. Glob Health. 2006;2:6.

17. Haque KN. Definitions of bloodstream infection in the newborn. Pediatr Crit Care Med. 2005;6:545-9.

18. Bizzarro MJ, Raskind C, Baltimore RS, Gallagher PG. Seventy-five years of neonatal sepsis at Yale: 1928-2003. Pediatrics. 2005;116:595-602.

19. Gladstone IM, Ehrenkranz RA, Edberg SC, Baltimore RS. A ten-year review of neonatal sepsis and comparison with the previous fifty-year experience. Pediatr Infect Dis J. 1990;9:819-25.

20. Hammoud MS, Al-Taiar A, Al-Abdi SY, Bozaid H, Khan A, AlMuhairi LM, Rehman MU. Late-onset neonatal sepsis in Arab states in the gulf region: two-year prospective study. Int J Infect Dis. 2017;55:125-30.

21. Bizzarro MJ, Shabanova V, Baltimore RS, Dembry LM, Ehrenkranz RA, Gallagher PG. Neonatal sepsis 2004-2013: the rise and fall of coagulasenegative staphylococci. J Pediatr. 2015;166:1193-9.

22. Zaidi AK, Huskins WC, Thaver D, Bhutta ZA, Abbas Z, Goldmann DA Hospital-acquired neonatal infections in developing countries. Lancet. 2005; 365:1175-88.

23. Saleem AF, Qamar FN, Shahzad H, Qadir M, Zaidi AK. Trends in antibiotic susceptibility and incidence of late-onset klebsiella pneumoniae neonatal sepsis over a six-year period in a neonatal intensive care unit in Karachi, Pakistan. Int J Infect Dis. 2013;17:e961-5.

24. Guo J, Luo Y, Wu Y, Lai W, Mu X. Clinical characteristic and pathogen spectrum of neonatal sepsis in Guangzhou city from june 2011 to june 2017. Med Sci Monit. 2019;25:2296-304.

25. Li X, Ding X, Shi P, Zhu Y, Huang Y, Li Q, Lu J, et al. Clinical features and antimicrobial susceptibility profiles of culture-proven neonatal sepsis in a tertiary children's hospital, 2013 to 2017. Medicine (Baltimore). 2019;98: e14686.

26. Marchant EA, Boyce GK, Sadarangani M, Lavoie PM. Neonatal sepsis due to coagulase-negative staphylococci. Clin Dev Immunol. 2013;2013:586076.

27. Shah AJ, Mulla SA, Revdiwala SB. Neonatal sepsis: high antibiotic resistance of the bacterial pathogens in a neonatal intensive care unit of a tertiary care hospital. J Clin Neonatol. 2012;1:72-5.

28. Afsharpaiman S, Torkaman M, Saburi A, Farzaampur A, Amirsalari S, Kavehmanesh Z. Trends in incidence of neonatal sepsis and antibiotic susceptibility of causative agents in two neonatal intensive care units in Tehran, i. R iran J Clin Neonatol. 2012;1:124-30.

29. Pammi M, Holland L, Butler G, Gacser A, Bliss JM. Candida parapsilosis is a significant neonatal pathogen: a systematic review and meta-analysis. Pediatr Infect Dis J. 2013;32:e206-16.

30. Garges HP, Moody MA, Cotten CM, Smith PB, Tiffany KF, Lenfestey R, Li JS, et al. Neonatal meningitis: what is the correlation among cerebrospinal fluid cultures, blood cultures, and cerebrospinal fluid parameters? Pediatrics. 2006:117:1094-100.

31. Hoque MM, Ahmed AS, Chowdhury MA, Darmstadt GL, Saha SK. Septicemic neonates without lumbar puncture: what are we missing? J Trop Pediatr. 2006;52:63-5.

32. Ballot DE, Bandini R, Nana T, Bosman N, Thomas T, Davies VA, Cooper PA et al. A review of -multidrug-resistant enterobacteriaceae in a neonatal unit in johannesburg, South Africa. BMC Pediatr. 2019;19:320.

33. Goll C, Balmer P, Schwab F, Ruden H, Eckmanns T. Different trends of mrsa and vre in a german hospital, 1999-2005. Infection. 2007;35:245-9.

34. Astani A, Zimmermann S, Hassan E, Reichling J, Sensch KH, Schnitzler P. Antimicrobial activity of propolis special extract gh 2002 against multidrugresistant clinical isolates. Pharmazie. 2013:68:695-701.

35. Chiu CH, Michelow IC, Cronin J, Ringer SA, Ferris TG, Puopolo KM. Effectiveness of a guideline to reduce vancomycin use in the neonatal intensive care unit. Pediatr Infect Dis J. 2011;30:273-8.

36. Kaufman DA. "less is more": decreasing antibiotic days in the nicu. J Pediatr. 2013:89:424-5.

\section{Publisher's Note}

Springer Nature remains neutral with regard to jurisdictional claims in published maps and institutional affiliations. 